\title{
On the mechanical behaviour of a crystalline undulator
}

\begin{abstract}
A crystalline undulator is a newly proposed compact device to generate coherent electro-magnetic waves. The crystalline undulator is a micro-electro-mechanical system that relies on silicon micro-fabrication. In the present work, the mechanical behaviour of a crystalline undulator with sub-millimetric period obtained by patterning the surfaces of a silicon lamina with alternate strips has been investigated. The anisotropy of the silicon substrate and the geometrically nonlinear behaviour of the device have been discussed. Sensitivity study has been performed for defining the optimal thickness and width of the strips. Finally, an analytical model is proposed to evaluate the displacements and local effects such as interfacial shear stress concentrations.
\end{abstract}

Keywords: crystalline undulator; channelling; residual stress; numerical simulations; analytical models.

\section{Introduction}

An undulator is a device to generate coherent electromagnetic radiation across the UV and x-ray ranges. Electrical charges are forced to oscillate in an electromagnetic field of period $L$ and emit electromagnetic (e.m.) radiation due to acceleration (bremstrahlung). Propagation of e.m. radiation generated at one oscillation yields stimulated emission at next oscillation, i.e., coherent superposition of e.m. waves occurs, leading to emission of monoenergetic, coherent, collimated and intense radiation (Figure 1). This is the working principle of the free electron laser (FEL) [1], whose operation has been experimentally demonstrated at some laboratories worldwide down to $L \cong 1 \mathrm{~cm}$. The spectrum of e.m. radiation peaks approximately at the frequency $v=2 c \gamma^{2} / L$, where $c$ is the light speed and $\gamma$ the relativistic factor [2]. In modern accelerators $\gamma$ is of the order of $10^{3}$.

Channeling is the confinement of positively charged particles in the electromagnetic field of a crystalline lattice [3]. Channeling occurs as the particle's momentum is nearly parallel to a crystalline plane (planar channeling) or major lattice direction (axial 
channeling). Thereby, a periodically undulated crystal would create a preferential pathway for positively-charged particles under channeling condition (Figure 1).

Undulation radiation (UR) is the result of charge oscillations in the crystal [4-6], whose period lies in the millimetric or sub-millimetric range, i.e., a periodicity which is not currently accessible to FEL. Preliminary indication of the achievement of UR has been recently reported [7] though the search for clearer experimental evidences is still under investigation [8].

Various methods have been proposed to realize a crystalline undulator (CU), many of which are summarized in Ref.[9]. As suggested in Ref. [10], a promising technique for the realization of a CU takes advantage of modern technology in microelectronics, about patterning of a silicon substrate with deposition of a thin layer of insulator onto its surface. Indeed, an alternate series of thin tensile strips deposited on both sides of a silicon wafer and perpendicularly to the nominal trajectory of charged particles, would generate a periodic deformation field. Channeling of particles in a periodically deformed lattice guarantees oscillations and, in turn, e.m. radiation emission. An investigation to show that the proposed method matches the requirements for UR emission has recently appeared in the literature [9].

CU may be used to generate hard x-ray radiation, whose properties are fundamental to high-resolution crystallography. Such technique will allow precise determination of reaction mechanism of most biological systems, a knowledge which is poorly accessible with currently available techniques.

Although rather a clear understanding of the physical processes involved in the fabrication of a $\mathrm{CU}$ does exist, the need for an engineering investigation is required with the aim to aid further development and to implement future fabrication of the device.

The paper is organized as follows. The main aspects of the object of study and the description of the problem are given in section 2; subsequently, a brief description of the procedure performed to realize the undulator is briefly given in section 2.1 . The choice of the mechanical parameters employed in the numerical simulations is discussed in section 2.2, whereas the assumptions concerning the mechanical models are presented in section 2.3; in this section the principal results obtained from the numerical analysis are also compared with theoretical and experimental data in order to validate the finite element model employed. In section 3 the results of the numerical simulations of the undulator are reported. In order to evaluate the deformation of the structure at hand, silicon substrate can be modelled like an isotropic material without appreciable 
errors: this is showed in section 3.1. To assess the effects of the design parameters on the mechanical behaviour of the undulator, a sensitivity study is performed in section 3.2; next, a simplified method utilizable to predict average amplitude of the periodic undulation is proposed in section 3.3. Local effects, such as stress concentrations, are also evaluated with an analytical formulation proposed by one of the authors in section 3.4 and compared with numerical solutions.

\section{Description of the problem}

The object of the present study is the mechanical behaviour of a crystalline undulator (CU) with sub-millimetric period, obtained patterning the surface of a plane silicon (Si) lamina with strips in silicon nitride $\left(\mathrm{Si}_{3} \mathrm{~N}_{4}\right)$. The alternate strips (Figure 2) are applied on the substrate in order to obtain a periodic undulation of the lamina.

At the Sensors and Semiconductors laboratory of the University of Ferrara, crystalline silicon undulators having various substrate thickness have been realized through LPCVD method (see section 2.2) applied to circular silicon wafers [10]. Previous experiences revealed that $200 \mu \mathrm{m}$ is a convenient value of the substrate thickness in order to obtain appropriate values of deformation amplitudes and radii of curvature suitable to realizing a CU with sub-millimetric undulation. Thus, in the present study, $h_{s}$ is assumed equal to $200 \mu \mathrm{m}$.

In order to obtain a sub-millimetric periodicity of the deformation, strips width $a$ and strips spacing $p$ are assumed in the range 100-500 $\mu \mathrm{m}$. Accordingly, the value of film thickness $h_{f}$ is of the order of 100-1000 nm.

To avoid de-channeling of particles [11], previous experience suggests to assume the length $l$ of the undulator equals to some millimetres; moreover, the width $w$ of the undulator is dictated by sake of practice to be equal to few centimetres.

The periodic undulations are obtained inducing residual stress which arise from the deposition process of the coating [12].

The aim of the present work is to study the feasibility of CU having the above characteristics and to evaluate optimal thickness $h_{f}$ and width $a$ of $\mathrm{Si}_{3} \mathrm{~N}_{4}$ strips in order to obtain a periodic deformation of the system with amplitude of approximately $3 \mathrm{~nm}$. This value of the transverse amplitude for the substrate displacements would render the undulator utilizable to obtain coherent radiation through channelling phenomenon [13]. 
Another important parameter which must be taken into consideration for correct design of a $\mathrm{CU}$ is the radius of curvature. According to the theory, channelling is prevented below an energy-dependent critical radius [11].

\subsection{Fabrication technology}

In the present work a periodic deflection of a crystalline substrate is obtained patterning its surfaces with alternate strips [9] through a low pressure chemical vapour deposition (LPCVD) technique. The advantages concerning this method have been reported previously [14].

In the following, a brief description of the procedure to realize the undulator is given. For more details concerning CVD technique see, for example, Ref. [15].

A circular silicon wafer with orientation (110), having diameter of 4 in and average thickness $h_{s}$ of $200 \mu \mathrm{m}$ (Figure 3) was introduced in a furnace at the temperature of $600^{\circ} \mathrm{C}$, suitable for LPCVD.

After that low pressure (200 millitor) is made in the furnace, the temperature is increased until $900^{\circ} \mathrm{C}$ and vapours of nitrogen protoxide $\left(\mathrm{N}_{2} \mathrm{O}\right)$ and dichlorosilane $\left(\mathrm{SiH}_{2} \mathrm{Cl}_{2}\right)$ are fluxed to obtain silicon oxide $\left(\mathrm{SiO}_{2}\right)$ : the gas fluxes are dosed in order to obtain an oxide deposition of few nanometers on the wafer surfaces. Subsequently, temperature is decreased to $750^{\circ} \mathrm{C}$; at this point ammonia $\left(\mathrm{NH}_{3}\right)$ and dichlorosilane were fluxed in the furnace to obtain silicon nitride $\left(\mathrm{Si}_{3} \mathrm{~N}_{4}\right)$.

Once that the silicon nitride deposition has been completed, the temperature is increased until $900^{\circ} \mathrm{C}$ and a second $\mathrm{SiO}_{2}$ deposition is made on the $\mathrm{Si}_{3} \mathrm{~N}_{4}$ film; following the wafer comes cooled.

Deposition of a $\mathrm{SiO}_{2}$ layer is made to improve the adhesion between the film and the substrate: in a preliminary study, we noticed that silicon nitride layers having thickness of 50-200 nm, deposited on silicon wafers without previous deposition of $\mathrm{SiO}_{2}$ film, delaminated some months later the deposition. In effects, it has been showed that a gradual spatial variation of the mechanical properties in compositionally graded or multilayered structures can reduce the residual stress, the concentrations of stress at the corners and in correspondence of the edges, and, consequently, the probability of delamination and cracks propagation, improving the strength and the stability of these kind of structures in the time. This is a promising field of application of the functionally graded materials (e.g. [16-18]). 
In order to realize the patterning of the surfaces of the wafer, few microns of a fluid polymeric photoresist (SHIPLEY S1813) is deposited on both the surfaces and fixed through an hot plate at $115^{\circ} \mathrm{C}$. A photolithographic glass mask comes superposed on the wafer and exposed to UV radiation which make the impression of the photoresist: this technique is applied on both sides of the wafer so as to obtain an alternate disposition of the patterns. The wafer is now dipped in a development solution for 45-60 seconds to remove the impressed photoresist obtaining a patterned surface. To remove the external $\mathrm{SiO}_{2}$ layer from the exposed zones, the wafer is dipped in hydrofluoric acid (HF) solution buffered with an ammonium fluoride $\left(\mathrm{NH}_{4} \mathrm{~F}\right)$ solution obtaining alternate $\mathrm{Si}_{3} \mathrm{~N}_{4}$ surfaces. Next, the wafer is immersed in phosphoric acid $\left(\mathrm{H}_{3} \mathrm{PO}_{4}\right)$ solution heated at $120^{\circ} \mathrm{C}$ to remove the $\mathrm{Si}_{3} \mathrm{~N}_{4}$ film using silicon oxide as masking layer; the residual silicon oxide layer is removed with BHF solution. This produces a periodic deflection of the lamina due to residual stress exchanged across the contact surface between the strips and the wafer. Finally, cutting properly the wafer with a diamond circular saw, the undulator is obtained.

\subsection{Mechanical properties}

As known, crystalline silicon is an anisotropic material having face-centered cubic symmetry, consequently its behaviour can be descript by only 3 material constants, which dependence of from the crystallographic directions has been investigated $[19,20]$.

For example, using Voigt notation to write constitutive equations $\sigma=C \varepsilon$, $\sigma=\left\{\begin{array}{llllll}\sigma_{11} & \sigma_{22} & \sigma_{33} & \sigma_{23} & \sigma_{13} & \sigma_{12}\end{array}\right\}$ and $\varepsilon=\left\{\begin{array}{llllll}\varepsilon_{11} & \varepsilon_{22} & \varepsilon_{33} & 2 \varepsilon_{23} & 2 \varepsilon_{13} & 2 \varepsilon_{12}\end{array}\right\}^{T}$ being the stress tensor and the infinitesimal strain tensor respectively, the elastic stiffness tensor $\boldsymbol{C}$ can be expressed in matrix form, which in (100) plane assumes, in average, the values [12]:

$$
\boldsymbol{C}^{(100)}=\left(\begin{array}{cccccc}
166.2 & 64.4 & 64.4 & 0 & 0 & 0 \\
64.4 & 166.2 & 64.4 & 0 & 0 & 0 \\
64.4 & 64.4 & 166.2 & 0 & 0 & 0 \\
0 & 0 & 0 & 79.8 & 0 & 0 \\
0 & 0 & 0 & 0 & 79.8 & 0 \\
0 & 0 & 0 & 0 & 0 & 79.8
\end{array}\right) \mathrm{GPa}
$$

Elastic stiffness matrix relative to a generic crystallographic direction (Figure 4) can be obtained applying the transformation rule for basis changes. 
In case at hand, the wafer surface is parallel to the (110) plane, and the correspondent matrix $\mathrm{C}$ results:

$$
\boldsymbol{C}^{(110)}=\left(\begin{array}{cccccc}
195.1 & 35.5 & 64.4 & 0 & 0 & 0 \\
35.5 & 195.1 & 64.4 & 0 & 0 & 0 \\
64.4 & 64.4 & 166.2 & 0 & 0 & 0 \\
0 & 0 & 0 & 79.8 & 0 & 0 \\
0 & 0 & 0 & 0 & 79.8 & 0 \\
0 & 0 & 0 & 0 & 0 & 50.9
\end{array}\right) \mathrm{GPa}
$$

Young modulus and the Poisson ratios along the principal directions contained into a plane parallel at wafer surface can be calculated:

$E_{[110]} \cong 170 \mathrm{GPa} ; \quad E_{[-110]} \cong 170 \mathrm{GPa} ; \quad E_{[001]} \cong 130 \mathrm{GPa} ;$

$v_{[-110]}^{[110]} \cong 0.062 ; \quad v_{[-110]}^{[001]} \cong 0.363 ; \quad v_{[001]}^{[-110]}=v_{[001]}^{[110]} \cong 0.279 ;$

The strips are patterned on the wafer surface parallel to the [-110] direction.

To evaluate the influence of anisotropy on the results, numerical simulations have been done considering both the stiffness matrix $C^{(110)}$ and average values of Young modulus $E_{[-110]}$ and Poisson ratio $v_{[-110]}^{[110]}$ for an hyperelastic isotropic material. In section 3 it will be showed that the anisotropy does not affect significantly the results.

Moreover, at the laboratory of polymeric materials of the Department of Engineering of Ferrara, tensile tests and three-point bending tests have been performed on specimens having the dimension of $60 \times 10 \times 1 \mathrm{~mm}$, obtained by cutting properly silicon wafers, with the aim of evaluating the Young modulus of the silicon along the orthogonal directions [001] and [-110].

The material testing machine, having a capacity of $500 \mathrm{~N}$, was connected to a computer system to acquire and record the applied load and the displacements of the specimens. The results obtained from the mechanical tests were in good agreement with the values of Young modulus of the silicon reported in literature.

For $\mathrm{Si}_{3} \mathrm{~N}_{4}$, deposition to achieve films with internal stress occurs under non-standard conditions; for the purpose, non-stoichiometric proportion between $\mathrm{Si}_{3} \mathrm{~N}_{4}$ are used. Based on previous knowledge, we assume the following average parameters:

Si substrate: $\quad E_{s}=170 \mathrm{GPa} ; \quad v_{s}=0.062$;

$\mathrm{Si}_{3} \mathrm{~N}_{4}$ film: $\quad E_{f}=310 \mathrm{GPa} ; v_{f}=0.24$;

where $E$ denotes the Young modulus and $v$ the Poisson ratio. All these quantities are considered temperature independent. 


\subsection{Mechanical models}

Finite element models have been employed to simulate the behaviour of a circular silicon wafers uniformly covered by a $\mathrm{Si}_{3} \mathrm{~N}_{4}$ film of different thickness.

It has been shown that the residual stress $\sigma_{f}$ in the film deposited via LPCVD technique does not depend appreciably on the film and substrate thickness, and in the case at hand it turns out to be $1.2 \mathrm{GPa}$.

The simplest way to simulate the various residual effects induced by intrinsic and extrinsic stresses growth during to the process of film deposition consists to submitting the modelled wafer only to an equivalent thermal load $\Delta \mathrm{T}$ such as to produce the same stress in the coating [21].

The magnitude of the equivalent thermal load can be calculated through the expression:

$\Delta \mathrm{T} \cong \sigma_{f} /\left\{\left[\left(E_{f} /\left(1-v_{f}\right)\right]\left(\alpha_{s}-\alpha_{f}\right)\right\}\right.$, where $\alpha_{s}, \alpha_{f}$ represent the coefficients of thermal expansion of the silicon and silicon nitride respectively [22]. Being $\alpha_{s}=310^{-6}{ }^{\circ} \mathrm{C}^{-1}, \alpha_{f}=$ $110^{-6}{ }^{\circ} \mathrm{C}^{-1}$, it results $\Delta \mathrm{T} \cong 1500{ }^{\circ} \mathrm{C}$.

Numerical simulations were made using STRAUS7 (release 2.3.3) finite element analysis system [23]. The wafer is considered simply supported at the centre; the $\mathrm{Si}_{3} \mathrm{~N}_{4}$ film is modelled by 2D membrane elements and it is rigidly linked at the silicon substrate which is modelled by plate and shell elements. Both film and substrate layer are simulated by 456 finite elements (Figure 5).

In Figure 6 the deformed shapes of the midplane of the wafer substrate having $h_{s}=$ $200 \mu \mathrm{m}$ and $h_{f}=400 \mathrm{~nm}$ is reported as dotted line. Let us observe that the boundary displacement provided by the geometrically linear analysis, equals to $0.53 \mathrm{~mm}$, is about the triple of the substrate thickness $h_{s}$.

Nonlinear deformation theory should be used when plate deflections are no longer small compared to the plate thickness [24]. Moreover, the normalized mismatch strain exceeds the approximate value of 0.3 suggested by Freund [25] as a limit of validity of linear kinematics.

A geometrically nonlinear analysis has been performed for the wafer at hand, and the solid line in Figure 5 represents the deformed shape of the wafer. From this analysis it has been obtained a boundary displacement equal to $0.3 \mathrm{~mm}$.

However, it must be remarked that the object of the present study is a lamina partially covered by strips as depicted in Figure 2, where the width of the strips is of the order of some hundreds of microns. For this structure, the strain at the midplane 
substrate induced by deflection is much smaller than the substrate thickness, and linear kinematics can be adopted.

To validate the numerical results, the values of radii of curvature $R$ and the film stress $\sigma_{f}$ obtained from the simulations have been compared with experimental data and with the values of stress predicted by Stoney law [26]:

$\sigma_{f}=\frac{\bar{E}_{s} h_{s}^{2}}{6 R h_{f}}$

$\bar{E}_{s}=E_{s} /\left(1-v_{s}\right)$ or $\bar{E}_{s}=E_{s} /\left(1-v_{s}\right)^{2}$ being for either plane stress or plane strain, respectively, with obvious significance of the values in the proper context. The results are reported in Table I for the values of thickness of the substrate and film indicated.

The radii of curvature have been measured through a He-Ne collimated laser beam: laser is reflected from a point of the wafer surface, then while laser source is kept fixed, the wafer is moved and the laser beam is reflected at a different point on the wafer surface, so that the reflected point changes its position. Measuring the laser-to-wafer distance and the displacement of reflected point it is possible to calculate the radius of curvature of the wafer.

As known, Stoney law (1) has been widely employed for stress-strain analysis in thin films science because its simplicity and to the advantage that it does not involve the mechanical properties of the coating.

Nevertheless, it should be noted that (1) holds for homogeneous and isotropic materials, neglecting the contribute of the stiffness of the coating on the substrate strain; moreover, small components of the strain and displacement field and spatially uniform curvature are implicitly assumed.

The film stiffness is negligible in the cases in which it is $E_{f} h_{f}<<E_{s} h_{s}$; however, the thickness of the coating can be taken into account by means of different methods [2730]. Freund and Suresh [12] have proposed a correction term to evaluate the influence of the film thickness: in case at hand this term is comprised in the range $1-4 \%$, thus the film can be considered thin respect to the substrate, and regarding to this aspect, Stoney law can be applied without significant errors.

Another aspect concerns the homogeneity and the isotropy of the materials employed to realize the undulator. The technology of production previously described assures that the silicon substrate can be assumed homogeneous; possible non-homogeneities are due to the presence of the layer of silicon oxide $\mathrm{SiO}_{2}$ between the substrate and the coating, and to the variations of the mechanical properties of the thin film in its thickness. These 
variations can be highly influenced from many factors characterizing the development of deposition, the most important of which are the substrate temperature and the rate of the chemical vapour fluxes $[12,15,31-33]$. The $\mathrm{SiO}_{2}$ layer is very thin (few nanometers) compared to the film thickness, and as a matter of fact, it does not influence the mechanical behaviour of the structure.

$\mathrm{Si}_{3} \mathrm{~N}_{4}$ deposited via LPCVD can be considered as amorphous and consequently isotropic. If the film is very thin (i.e. $h_{s}<<h_{f}$ ), as discussed in $[12,30,34]$ for linearly elastic materials, the influence of non-homogeneities can be neglected, and average mechanical properties for the coating can be adopted.

Indeed, from the results reported in Table I, one can observe a good agreement between the values furnished by numerical analysis and experimental and theoretical data; this probes the substantial correctness of the previous assumptions.

\section{Undulator}

In the present section, the main results obtained from numerical simulations of the crystalline undulator (Figure 2) are presented. The objective of the analysis is to assess the values of the average amplitudes and radii of curvature of the periodic undulation of the system varying the geometric parameters $a, h_{f}, p$. At the Department of Physics of the University of Ferrara, the design of undulators with 10 strips on one side and 9 strips on the other has been developed; a transversal section of the structure is depicted in Figure 7a. However, also a more simple design has been considered, as it is shown in Figure $7 \mathrm{~b}$. Because its simplicity, mechanical behaviour of this last structure is studied in some detail in order to assess the effects of silicon anisotropy and to compare numerical results with those obtained by an analytical formulation proposed by the first author (see section 3.4).

\subsection{FEM analysis}


The silicon substrate and the strips are modelled assuming plane strain conditions. The structure is simply supported at the ends and it is subjected to a thermal load as discussed in section 2.3.

Amplitudes of the transversal displacements of the medium plane of the substrate are reported in Figure 8a and $8 \mathrm{~b}$ for $h_{s}=0.2 \mathrm{~mm}, h_{f}=400 \mathrm{~nm}, a=0.250 \mathrm{~mm}, p=0.500$ $\mathrm{mm}$; silicon has been modelled as an isotropic material assuming the average values $E_{s}$ $=E_{[001]}=170 \mathrm{GPa}$ and $v_{s}=v_{[001]}^{[110]}=0.062$.

In order to evaluate the influence of the anisotropy of the silicon, numerical simulations of the undulator $3+2$ have been done using both the stiffness matrix $C^{(110)}$ (see section 2.2) and average values $E_{s}=E_{[001]}$ and $v_{s}=v_{[001]}^{[110]}$ for an isotropic material.

The results in terms of transversal displacements of the midplane and upper surface of the lamina are reported in Figure 9a and 9b respectively, for $h_{s}=0.2 \mathrm{~mm}, h_{f}=400$ $\mathrm{nm}, a=0.250 \mathrm{~mm}, p=0.500 \mathrm{~mm}$ : let us note that differences concerning the deformation amplitude and osculating circle radii are lower than $5 \%$, and this difference for the undulator $10+9$ is furtherly reduced to less than $1 \%$. It can be concluded that, in order to evaluate displacements field of the structure, the silicon can be considered isotropic without large errors. Under this assumption, a simpler analytical model to study the mechanical behaviour of the system at hand, in particularly to evaluate locally the stress field, can be developed.

\subsection{Sensitivity analysis}

In order to show the response of the undulator $10+9$ of Figure 7 a varying the design parameters, in the following some results obtained from a sensitivity study are reported.

The values of average amplitude deformation and osculating circle radii of the substrate midplane are reported in Table II for $h_{s}=0.2 \mathrm{~mm}, h_{f}=200 \mathrm{~nm}$ and $400 \mathrm{~nm}$, strip width $a=0.250 \mathrm{~mm}$, varying the strip spacing $p$. It should be noted that the radii of curvature obtained for $h_{f}=200 \mathrm{~nm}$ are almost the double of those obtained for $h_{f}=400$ $\mathrm{nm}$, as predicted by Stoney law for a constant value of $\sigma_{f}$; moreover, for $h_{f}=400 \mathrm{~nm}, a$ $=250 \mathrm{~nm}$ and $p=0.5 \mathrm{~mm}$, it has been found an average amplitude near $3 \mathrm{~nm}$. As explained previously, this value would be render the undulator utilizable to obtain coherent emissions. Numerical analysis have been performed to evaluate also the effects 
of strip width on the displacements field of the structure: the results obtained are reported in Table III for $h_{s}=0.2 \mathrm{~mm}, h_{f}=400 \mathrm{~nm}$ and constant spacing $p=0.250 \mathrm{~mm}$. As expected, for $a=p / 2$ the displacements amplitude reaches the maximum value.

Transversal displacements of the order of $3 \mathrm{~nm}$ can be obtained decreasing the strip spacing and increasing the film thickness. For example, this is showed in Table IV for $h_{s}=0.2 \mathrm{~mm}, h_{f}=400 \mathrm{~nm}$ and strip spacing $p=0.250 \mathrm{~mm}$. As before, in the case in which $a=p / 2$, the displacements amplitude reaches the maximum value, in the present case equals to $3.2 \mathrm{~nm}$.

From the results above, both the following series of geometric parameters $h_{f}=400$ $\mathrm{nm}, a=0.250 \mathrm{~mm}, p=500 \mathrm{~mm}$, or $h_{f}=1600 \mathrm{~nm}, a=0.125 \mathrm{~mm}, p=250 \mathrm{~mm}$ are appropriate to realize a crystalline undulator. Finally, the following design parameters are assumed: $h_{s}=0.2 \mathrm{~mm}, h_{f}=400 \mathrm{~nm}, a=0.250 \mathrm{~mm}$ and $p=0.500 \mathrm{~mm}$. This choice is justified by a better homogeneity in the coating at relatively thin layers, hence, for this reason undulators with $h_{f}=1600 \mathrm{~nm}$ are not considered.

\subsection{A simplified analysis model}

The deformed shape of the undulator 10+9 (Figure 8a) suggests a very simple method to evaluate roughly the average amplitude of periodic undulation in case in which the strips do not overlap, i.e. for $p \geq 2 a$, based on elementary structural mechanics.

The deformation of the undulator can be approximated considering a beam of length equals to $p / 2$, having a moment of inertia $J_{s}=h_{s}{ }^{3} / 12$, simply supported at the ends and loaded by two couples applied in correspondence of the ends of the cover, as reported in Figure 10. The amplitude deformation $f$ can be calculated through following elementary formula:

$$
f=\frac{C a}{8 \bar{E}_{s} J_{s}}(p-a)
$$

$C=\sigma_{f} h_{f} h_{s} / 2$ being the couples transmitted to the substrate from the strip.

Applying formula (2) for $h_{s}=0.2 \mathrm{~mm}, h_{f}=400 \mathrm{~nm}, p=0.500 \mathrm{~mm}$ and $a=0.250 \mathrm{~mm}$ one obtain $f \cong 2.76 \mathrm{~nm}$, and the difference with respect to the value furnished by finite elements model is less than $10 \%$. 


\subsection{Local analysis}

Delamination, failures and crack formations in film-substrate systems can occur due to the high residual stresses generated during the deposition process of the coating on the substrate [35].

These phenomena and the possible loss of adhesion of the strips from the substrate may compromise completely the functionality of the devices constituted by micro-layered structures (see section 2.1).

As well known, the points in which the stress reaches the maximum intensities are the most probable locations of crack initiation and growth; for this reason, locations and nature of stress singularities are fundamental subjects in fracture mechanics.

FEM analysis or, more suitably, analytical models can be employed to investigate stress concentrations.

The contact problem of a thin cover or periodically spaced thin strips applied to an elastic half space has been investigated from many authors (e.g. [36-39]). In these references, as in the case in object, a very thin film is considered; this makes it possible to assume that only shear stress along the contact region is transmitted. The Green's function for the half plane subjected to a unit tangential load (i.e. the Flamant solution), is used to define the strain of the half plane, and the condition of perfect adhesion between the stiffener and the half plane leads to a singular integral equation which can be solved using different approaches. As reported in [38,39], the problem can be solved by expressing the interfacial shear stress as a series of orthogonal polynomials.

However, in the case at hand, the substrate has finite dimensions and Green's function is unknown; therefore, to study the mechanical behaviour of symmetric undulators, displacements field is approximated by means of a separation of variables representation through trigonometric functions along $x$ axis and exponential functions along $y$ axis [40]:

$$
u(x, y)=\sum_{n=1,3}^{\infty} U_{n}(y) \sin \left(\frac{n \pi x}{2 l}\right) ; \quad v(x, y)=\sum_{n=1,3}^{\infty} V_{n}(y) \cos \left(\frac{n \pi x}{2 l}\right)
$$

where: 
$U_{n}(y)=\left(A_{1}+A_{2} y / l\right) \sinh \left(\frac{n \pi y}{2 l}\right)+\left(A_{3}+A_{4} y / l\right) \cosh \left(\frac{n \pi y}{2 l}\right)$

$V_{n}(y)=\left(B_{1}+B_{2} y / l\right) \sinh \left(\frac{n \pi y}{2 l}\right)+\left(B_{3}+B_{4} y / l\right) \cosh \left(\frac{n \pi y}{2 l}\right) ;$

$U_{n}(y), V_{n}(y)$ being the amplitudes of horizontal and vertical displacements respectively, $l$ is the length of the lamina and $A_{n}, B_{n}$ are the integration constants.

The equilibrium equation for the strips and the condition of complete contact between the strips and the substrate surface leads to an algebraic system which can be solved in the unknown coefficients $C_{n}$ for the interfacial shear stress $\tau(x, 0)$, which is assumed in the form:

$$
\tau(x, 0)=\frac{E_{s}}{\sqrt{1-\left(\frac{x-p / 2}{a / 2}\right)^{2}}} \sum_{n=1,3}^{\infty} C_{n} T_{n}\left(\frac{x-p / 2}{a / 2}\right)
$$

$T_{n}(x)$ being the Chebyshev polynomials of the first kind.

The shear stress defined by (4) has a square root singularity in correspondence of the tips of the cover, i.e. for $x=p / 2 \pm a$.

The singular behaviour of the interfacial shear stress can be evaluated through the elastic shear stress singularity factor (SSSF, as called in [41]), which may be obtained through the following expression:

$\mathrm{SSSF}=\lim _{x \rightarrow p / 2 \pm a / 2} \sqrt{2 \pi\left[\frac{a}{2} \mp\left(x-\frac{p}{2}\right)\right]} \tau(x, 0)= \pm E_{s} \sqrt{\frac{a \pi}{2}} \sum_{n=1,3}^{\infty} C_{n}$

The analytic solution for an undulator with a given number of strips is obtained by superposing the solutions obtained for appropriate values of spacing $p$, and in the following, the results provided by the analytical model for the undulator $3+2$ (Figure 7b) having $h_{s}=0.2 \mathrm{~mm}, h_{f}=400 \mathrm{~nm}, p=0.500 \mathrm{~mm}$ and $a=0.250 \mathrm{~mm}$, are reported and compared with those obtained from numerical analysis. The results shown in figures are obtained taking a number of terms in the series equal to 100 .

Horizontal and vertical displacements of the surface and midplane substrate are reported in Figure 11 and 12; a good agreement between analytic and numerical solutions is showed. One can observe that the maximum difference between the solutions concerns the horizontal surface displacements, and it occurs in correspondence 
of the ends of the strips, since in these points the stress field predicted by analytical solution is unbounded.

In Figure 13a the horizontal stress along the thickness of the substrate is plotted. As shown, both analytic and numerical solutions predict an almost linear trend of the stress, as predicted by Stoney law (1). The interfacial shear stress is reported in Figure 13b. Significant differences between the analytic and FEM solutions can be observed near the tips of the strips; moreover, it has been noted that increasing the number of the terms of the series, an increase of the oscillation amplitudes occurs, as described by Gibbs phenomenon.

\section{Conclusions}

The mechanical behaviour of a crystalline undulator $(\mathrm{CU})$ with sub-millimetric period has been examined in details. Numerical analysis have been performed to evaluate the amplitude deformation of the system. In particular, the influence of the nonlinear geometric behaviour of the system and the anisotropy of the silicon substrate has been discussed and showed to be non relevant for the problem under study. A sensitivity analysis has been performed for definition of the optimal spacing, width and thickness of the strips. Moreover, the displacements and the interfacial shear stress concentration has been investigated by numerical and analytical methods.

In summary, an engineering approach to a specific physical problem of current interest has been addressed. The investigation has clarified the role of some assumptions in the approach to the problem and provides a valid tool for correct design of future CUs. 


\section{References}

[1] J.M.J. Madey, Stimulated emission of bremsstrahlung in a periodic magnetic field, J. App. Phys. 42 (1971) 1906.

[2] A.V. Korol, A.V. Solov'yov and W. Greiner, Int. J. Mod. Phys. E. 13 (2004) 867.

[3] A. F. Elishev, N.A. Filatova, V.M. Golovatyuk, I.M. Ivanchenko, R. B. Kadyrov, N.N. Karpenko, V.V. Korenkov, T.S. Nigmanov, V. D. Riabtsov, M.D.

Shafranov, B. Sitar, A.E. Senner, B.M. Starchenko, V.A. Sutulin, I.A. Tyapkin, E. N. Tsyganov, D.V. Uralsky, A.S. Vodopianov, A. Forycki, Z. Guzik, J.

Wojtkowska, R. Zelazny, I.A. Grishaev, G.D. Kovalenko, B.I. Shramenko, M.D. Bavizhev, N.K. Bulgakov, V.V. Avdeichikov, R.A. Carrigan, T.E. Toohig, W.M. Gibson, I.J. Kim, J. Phelps, C.R. Sun, Phys. Lett. B 88 (1979) 387.

[4] V.V. Kaplin, S.V. Plotnikov and S.A. Vorob'ev, Pis'ma Zh. Tekhn. Fiz. 50 (1980) 1079.

[5] V.V. Kaplin, S.V. Plotnikov and S.A. Vorob'ev, Abstracts of X-th Conf. on Appl. of Charged Particles for the study of Medium (1979) Moscow State Univ.

[6] A.V. Korol, A.V. Solov'yov and W. Greiner, Int. J. Mod. Phys. E. 8 (1999) 49.

[7] V.T. Baranov, S. Bellucci, V.M. Biryukov, G. I. Britvich, C. Balasubramanian, V. Guidi, G. Giannini, V.N. Zapolsky, V.I. Kotov, A.E. Kushnirenko, V.A.

Maisheev, G. Martinelli, E. Milan, V.A. Pikalov, V.I. Terekhov, U. Uggerhoj, V. N. Chepegin, Y.A. Chesnokov, J. Exp. Th. Phys. Lett. 82 (2005) 562.

[8] V.T. Baranov, V. Guidi, A. Antonini, E. Milan, A. Ronzoni, G. Martinelli, V.M. Biryukov, Y.A. Chesnokov, Nucl. Instrum. Meth. Phys. Res. B 252 (2006) 11.

[9] V. Guidi, L. Lanzoni, G. Martinelli, A. Mazzolari, A. Tralli, Design of a crystalline undulator based on patterning by tensile $\mathrm{Si}_{3} \mathrm{~N}_{4}$ strips on a $\mathrm{Si}$ crystal, Appl. Phys. Lett. 90 (2007) 114107.

[10] V. Guidi, A. Antonini, S. Baricordi, F. Logallo, C. Malagù, E. Milan, A. Ronzoni, M. Stefancich, G. Martinelli and A. Vomiero, Nucl. Instrum. Meth. Phys. Res. B 234 (2005) 40.

[11] V.M. Biryukov, Y.A. Chesnokov, V.I. Kotov, Crystal channelling and its application at high energy accelerators, Springer, Berlin, 1997.

[12] L.B. Freund, S. Suresh, Thin film materials. Stress, Defect formation and surface evolution. Cambridge University Press, 2003.

[13] S. Bellucci, V.M. Biryukov, G.I. Britvich, Y.A. Chesnokov, C. Balasubramanian, G. Giannini, V. Guidi,. Y.M. Ivanov, V.I. Kotov, V.A. Maisheev, C. Malagu, G. 
Martinelli, A.A. Petrunin, V.A. Pikalov, A. Raco, L. Silvi, V.V. Skorobogatov, M. Stefancich, F. Tombolini, D. Vincenzi, Phys. Rev. Spec. Topics - Accel. and Beams. 7 (2004) 023501-1.

[14] R.O.Avakian, K.T. Avetyan, K.A. Ispirian, E.G. Melikyan, Nucl. Instrum. Meth. Phys. Res. A 492 (2002) 11-13.

[15] M. Ohring, The Materials Science of thin films, Academic Press, 1992

[16] A.E. Giannakopoulos, S. Sureh, Indentations of solids with gradients in elastic properties : part I. Point force, International Journal of Solids and Structures 34(19) (1997) 2357-2392.

[17] S. Dag, W. F. Erdogan, A surface crack in a graded medium loaded by a sliding rigid stamp, Engineering Fracture Mechanics 69 (2002) 1729-1751.

[18] S. El-Borgi, W. Aloulou, A. Z.ghal, Buckling of a functionally graded coating with an embedded crack bonded to a homogeneous substrate, International Journal of Fracture 142 (2006) 137-150.

[19] J.J. Wortman, R.A. Evans, Young modulus, shear modulus, and Poisson's ratio in silicon and germanium, J. Appl. Phys. 36(1) (1965) 153-156.

[20] W.A. Brantley, Calculated elastic constants for stress associated with semiconductor devices, J. Appl. Phys. 44(1) (1973).

[21] I.D. Wolf, M. Ignat, G. Pozza, L. Minguet, H.E. Maes, J. Appl. Phys. 85 (2003) 6477.

[22] M. Stadtmüeller, Mechanical stress of CVD-Dielectrics, J. Electrochem. Soc. $139(12)(1992) 3669-3674$.

[23] Straus7 Theoretical Manual Software, 2004, $1^{\text {st }}$ edition.

[24] S.P. Timoshenko, S. Woinowsky-Krieger, Theory of Plates and Shells, McGrawHill $1959,2^{\text {nd }}$ edition.

[25] L.B. Freund, Substrate curvature due to thin film mismatch strain in the nonlinear deformation range, Journal of the Mechanics and Physics of Solids 48 (2000) 1159-1174.

[26] G.G. Stoney, Proc. R. Soc. London 9 (1909) 172-175.

[27] S.P. Timoshenko, Analysis of bi-metal thermostats, Journal of the Optical Society of America 11 (1925) 233-255.

[28] K.G. Soderberg, A.K. Graham, Proc. Am. Electro-platers' Soc. (1947) p.74 
[29] A. Brenner, S. Senderoff, Calculation of stress in electrodeposits from the curvature of a plated strip, Part of the Journal of Research of the National Bureau of Standards, Research paper RP1954 42 (1949) 105-123.

[30] L.B. Freund, Some elementary connections between curvature and mismatch strain in compositionally graded thin films, Journal of the Mechanics and Physics of Solids, 44(5) (1996) 723-736.

[31] O. Tabata, K. Kawahata, S. Sugiyama, I. Igarashi, Sensors and Actuators 20 (1989) 135-141.

[32] Y.C. Tai, R.S. Muller, Proc. of the $3^{\text {rd }}$ IEEE International Workshop on MicroElectro-Mechanical Systems (1990) 147-152.

[33] G.Coles, W.N.Shape Jr., R.L. Edwards, Proc. of the MEMS, Mechanical and Measurements Symposium (2001).

[34] F. Kroupa, Z. Knesl, J. Valach, Residual stresses in graded thick coatings. Acta Technica CSAV 38 (1993) 29-74.

[35] Z.C. Xia, J.W. Hutchinson, Crack patterns in thin films, Journal of the Mechanics and Physics of Solids 48 (2000) 1107-1131.

[36] N.Kh. Arutiunian, Contact Problem for a half-plane with elastic reinforcement, PMM 32(4) (1968) 632-646.

[37] N.Kh. Arutiunian, S.M. Mkhitarian, Periodic contact problem for a half-plane with elastic laps (cover plates), PMM 33(5) (1969) 813-843.

[38] G.A. Morar, G.Ia. Popov, On a contact problem for a half-plane with elastic coverings, PMM 35(1) (1971) 172-178.

[39] F. Erdogan, G.D. Gupta, The problem of an elastic stiffener bonded to a halfplane, ASME Journal of Applied Mechanics 38 (1971) 937-941.

[40] L. Lanzoni, E. Radi, On the problem of a coated elastic layer subjected to residual thermal stress; Proc. XVIII Congresso AIMeTA, Brescia, Italy, 2007.

[41] X.D. Wang, S.A. Meguid, On the electroelastic behaviour of a thin piezoelectric actuator attached to an infinite host structure, International Journal of Solids and Structures 37 (2000) 3231-3251. 
